\title{
Ovarian and cervical cancer
}

Chris Williams

This is the 5th in a series of articles examining recent developments in cancer
Royal South Hants Hospital, Southampton SO9 4PE

Chris Williams, consultant physician in medical oncology

Series edited by: Dr G M Mead.

BM于 1992;304:1501-4

\section{Ovarian carcinoma}

Ovarian carcinoma has been increasing in incidence and it is now the commonest malignancy of the female genital tract in most of the Western world. About 4500 women in England and Wales develop this cancer each year and there are 3700 deaths annually. The death rate of 14/100000 has doubled in the past 70 years even when standardised for age. Incidence rates for this tumour are low in Asia, Africa, and South America and are, in any individual country, higher in the professional classes. Despite these data suggesting environmental factors in its causation, little is known about them.

Risk of ovarian cancer is reduced by pregnancy and is highest in infertile women. Use of oral contraceptives also reduces the risk, though this may partly reflect the increased risk in infertile women. The lowest risk is, curiously, seen in women who have had tubal ligation. There is also a reduction in risk among women who have had a hysterectomy but whose ovaries were conserved. Despite this there has been a vigorous debate in the United States about whether women approaching the menopause who are undergoing a hysterectomy should also have a bilateral oophorectomy. ${ }^{1}$

Up to $5 \%$ of women who develop ovarian carcinoma have a strong family history of this tumour, and many of these families may have a genetic predisposition to developing ovarian, and sometimes other, cancers. ${ }^{23}$ Inheritance is probably autosomal dominant and researchers are close to identifying the gene(s) involved.

\section{DIAGNOSIS AND SURGERY}

Screening for ovarian cancer has been evaluated extensively in pilot studies. Although transvaginal ultrasonography with or without colour Doppler and screening for CA 125 have been shown capable of detecting "early" ovarian carcinoma, specificity remains an important problem. ${ }^{4}$ So far no studies have shown that early detection of ovarian cancer by screening improves survival, and its general use cannot be recommended. The United Kingdom Coordinating Committee for Cancer Research has proposed a national study of familial ovarian cancer, and this will offer screening to high risk family members.

Most patients with ovarian cancer have had nonspecific symptoms for some weeks or for a few months before diagnosis. Patients rarely present as surgical emergencies. So there is time to investigate and plan surgery before undertaking a laparotomy. This is important as surgery seems to be a keystone to prolonged survival. ${ }^{5}$ As well as carrying out thorough staging, current surgical recommendations include, where appropriate, an attempt at maximum debulking (residual tumour masses $<1.5 \mathrm{~cm}$ diameter) when complete clearance of tumours is not possible. This flies in the face of usual surgical tenets and surgeons not used to dealing with this tumour may fail to debulk even when this is possible. Despite lack of properly controlled trials testing the effectiveness of debulking surgery, current consensus suggests that debulked tumours are more responsive to chemotherapy and that patients with such tumours may survive longer and have better quality of life (fig 1 ). ${ }^{6}$ The development of chemotherapy capable of curing advanced disease is, however, likely to render surgical debulking obsolete.

\section{RADIOTHERAPY}

There is little evidence that radiotherapy has any role in managing ovarian cancer in women who have not had maximum debulking surgery or complete excision of the tumour. Thus any potential benefit may be in patients with "early stage" disease who have had optimal surgery. Even in these women there is debate about its effectiveness.

Studies into mechanisms of tumour spread (fig 2) and staging in the past two decades have clearly shown that the whole of the abdominal cavity is at risk of relapse in such women so pelvic irradiation alone is likely to prove inadequate. Unfortunately, only modest radiation doses can be delivered to the whole abdominal contents (2250 cGy in 30 fractions over four to six weeks, for example). And such doses may be inadequate to eliminate even microscopic metastases. Neverthe-

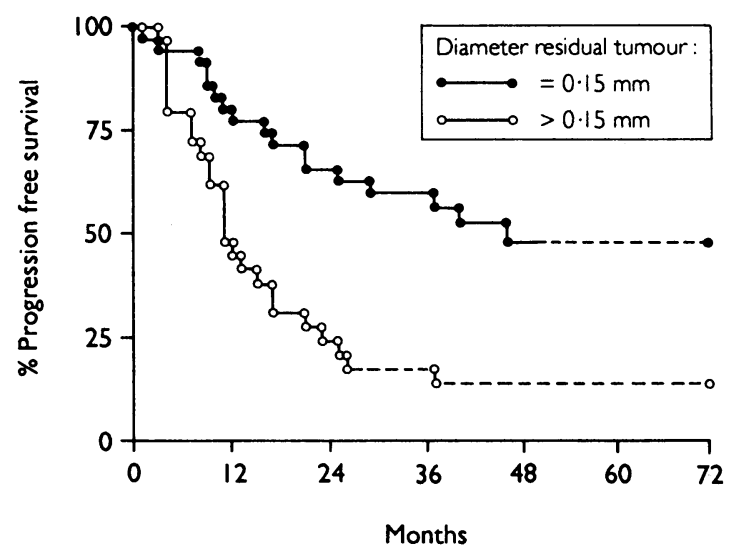

FIG 1-Relation between disease free survival after combination chemotherapy and diameter of largest tumour mass after primary cytoreductive surgery. ${ }^{\circ}$ Reproduced by permission of "Gynecologic Oncology," Academic Pres

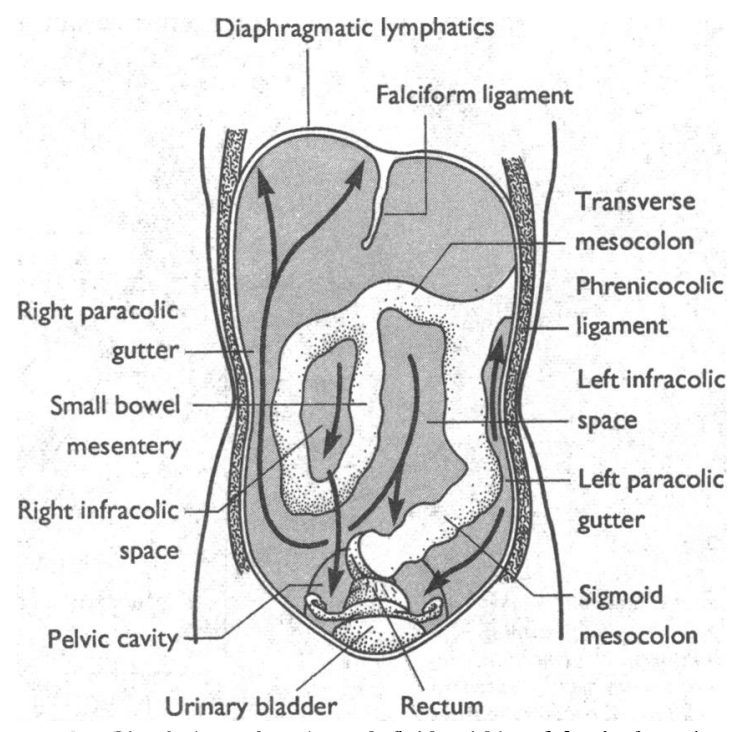

FIG 2-Circulation of peritoneal fluid within abdominal cavity. Reproduced by permission of Chapman and Hall 
TABLE I-Five year survival by stage in collected series of ovarian carcinoma ${ }^{\prime \prime}$

\begin{tabular}{lcc}
\hline & $\begin{array}{c}\text { No }(\%) \\
\text { of cases }\end{array}$ & $\begin{array}{c}\text { ative } \\
\text { at years }\end{array}$ \\
\hline Stage I & $2449(24)$ & 67 \\
Stage II & $1806(19)$ & 42 \\
Stage III and IV & $5540(57)$ & $14 \cdot 4$ \\
\hline
\end{tabular}

less, a series of randomised trials from Toronto have strongly suggested improved survival in women with stage I-III ovarian carcinoma undergoing optimal surgery who were treated with whole abdominal radiation compared with pelvic radiation with or without an alkylating drug. ${ }^{8}$ Similar randomised studies at the M D Anderson Hospital, however, have not supported these results." Smaller studies have also failed to show benefit for radiation. Currently, radiation is being little studied and is not used routinely by many centres. There may well still be a place, however, for well designed large trials testing its use as an adjuvant to surgery.

\section{CHEMOTHERAPY}

Ovarian cancer was one of the first tumours to be routinely treated with chemotherapy. Alkylating drugs rapidly became a standard treatment for advanced ovarian cancer and it is on these patients, the majority (table I), that most data are available. Numerous randomised clinical trials have tested the effectiveness of different types of chemotherapy in stage III and IV disease but there are few data comparing chemotherapy with a no treatment control. These studies have all been of modest size (50-300 patients) and it is therefore unsurprising that nearly all have failed to show significant differences between the treatments being tested. Despite this therapeutic fashions have evolved and changed over the past 15 years (box).

Because of the lack of reliable data the Medical Research Council in Britain organised a systematic overview of chemotherapy for advanced ovarian malignancy. This exhaustive meta-analysis of all the individual patient data from properly randomised trials, published and unpublished, comparing single drugs with combinations and assessing the role of platinum has recently been published."

Despite including over 8000 patients with advanced disease, of whom more than 6500 had died, this overview did not provide clear guidance about the best available current therapy. Rather it has defined questions suitable for large trials. Although little evidence exists that platinum prolongs survival when compared with an oral alkylating drug, platinum therapy is generally considered to be the first choice. This is based on its undoubtedly higher objective response rate, the likely consequent better symptom control, and the fact that many patients initially treated with an alkylating drug subsequently receive platinum on disease progression or relapse. Based on this, the finding that carboplatin is equally effective as cisplatin, and the suggestion that the addition of other drugs to platinum improves survival (fig 3), " a large scale trial for advanced ovarian carcinoma has begun. This

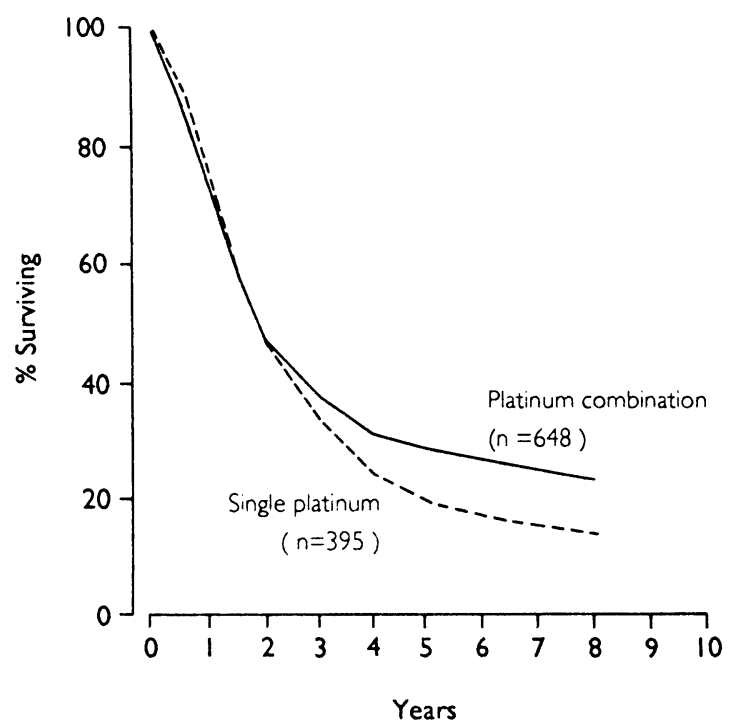

\section{Therapeutic fashions in chemotherapy of} advanced ovarian carcinoma

Before 1976 Single oral alkylating agent such as chlorambucil.

1976 Non-cisplatin based combination such as HEXACAF (hexamethylmelamine, cyclophosphamide, methotrexate, fluorouracil)

$\downarrow$

1980

Cisplatin based combination such as CAP (cyclophosphamide, doxorubicin, cisplatin)

$$
\downarrow
$$

Removal of doxorubicin from CAP type combinations leaving cyclophosphamide and cisplatin

Treatment with single agent cisplatin

$$
\downarrow
$$

1991 Substitution of carboplatin for cisplatin Overview suggests superiority of CAP over cisplatin alone. Further studies started.

study, sponsored by the Medical Research Council, compares a relatively non-toxic treatment carboplatin $\sigma$ with cisplatin, doxorubicin, and cyclophosphamide (CAP) to test whether there is a survival advantage for $\frac{\bar{D}}{\vec{D}}$ the more toxic combination.

Since the trials of adjuvant chemotherapy for "early" $\vec{\bullet}$ disease are too small a parallel trial is comparing? immediate platinum based chemotherapy with chemotherapy given at relapse. Both trials are being conducted by the International Collaborative Ovarian Neoplasm (ICON) Group. They are both international and the aim is to accrue 2000 patients in each trial.

\section{Summary}

The incidence of ovarian cancer continues to increase steadily and it now accounts for $1.3 \%$ of all deaths in women or $6 \%$ of deaths from cancer in women. No convincing aetiological factor has been identified. Debate still surrounds the concept of oophorectomy at $\frac{0}{3}$ the time of hysterectomy and an appropriately controlled trial is needed. Familial ovarian cancer may account for up to $5 \%$ of cases, and the gene(s) may soon be identified. Screening can be considered in family members but general population screening cannot be $\mathrm{N}$ recommended.

Radiotherapy is used rarely. When emploved whole abdominal fields should be used after removal of all or $N$ most of the tumour. The best chemotherapy regimen remains undefined despite numerous small trials. $\sigma$ Platinum drugs are generally accepted as the most active group of drugs and adding other drugs may further improve survival. This hypothesis is being $\mathscr{D}$ tested in the first large scale study of chemotherapy in 7 ovarian carcinoma.

\section{CARCINOMA OF THE CERVIX}

Invasive carcinoma of the cervix is the sixth commonest cancer in British women with an annual incidence of 16/100000 women. It accounts for about $4 \%$ of female malignancy and has a five year survival of $58 \%$, which is better than many other common产 cancers. Despite this there is a real need for improve-? ment in its management.

About $90 \%$ of invasive cervical tumours are squamous carcinomas, which usually arise from the squamo-columnar junction. The remainder are 
generally adenocarcinomas, arising from the endocervical glands in the endocervical mucosa, or mixed squamous adenocarcinomas. Since many tumours seem to have arisen after the initial development of a precursor premalignant condition (cervical intraepithelial neoplasia) screening for these lesions has been a cornerstone of attempts to reduce mortality.

The death rate from cervical cancer has fallen sharply in many developed countries: a phenomenon often explained by the introduction of screening, though this fall preceded the introduction of screening in many countries. Unfortunately, there were no randomised trials of cervical screening when it was first introduced so its contribution to falling mortality rates can be only guessed at. Despite this most workers in the subject support its use and the recent strategy document from the Department of Health The Health of the Nation, included targets for cervical cancer screening.

Unfortunately, the target is only that all women aged 20-64 years be invited for screening by the end of 1993 . This fails to overcome some of the problems associated with the cervical smear programme. The most important of these is that women at greatest risk of cervical cancer are the ones most likely to fail to accept an invitation for screening. At present $60 \%$ of women who develop cervical cancer have never been screened. Although general practitioners have been asked to fulfil screening targets in return for financial reward, inner city practices, where the incidence is highest, may see these targets as non-achievable and consequently be deterred from making a big effort. Help is needed in devising strategies which encourage women at risk to attend for screening.

A second problem is the adequacy of smears that are taken - any targets set should specify the rate of smears which are considered to be adequate for reporting. ${ }^{12}$ Inadequate smears cause anxiety for patients recalled, discourage future screening, and add to an already not inconsiderable cost. Changes in technique (Aylesbury spatula and cytobrush for instance) and improved technical competence should reduce rates of inadequate smears to about $5 \%$. Interpretation of cervical smears requires a high degree of skill, and audit systems should be mandatory in cytology units. However, even in the best laboratories the false negative rate may be as high as $10 \% .^{12}$

Because most cervical intraepithelial neoplasias evolve into invasive tumours only very slowly many clinicians have believed that follow up investigation of such lesions is not especially urgent. Because of pressure on colposcopy and other resources women in Britain often wait months before further investigation after an abnormal smear test result. This is unacceptable, not least because of the anxiety caused by such a delay but also because of the risk that there is already an invasive tumour-cytology may not detect concomitant invasive carcinomas. A prerequisite of any screening system is that a highly efficient mechanism is already in place to deal with women with positive screening test results. Cervical carcinoma fulfils most of the potential requirements for a successful screening programme - it remains to be seen whether there is the will to make it work. This means that any cervical screening programme must address the specific concerns of the women being screened, especially those at greatest risk.

\section{MANAGEMENT OF CERVICAL INTRAEPITHELIAL NEOPLASIA}

There remains much debate about the best way to care for women with cervical intraepithelial neoplasia. ${ }^{13}$ At least $50 \%$ of women with advanced lesions (grade III - severe dysplasia or carcinoma in situ) will go on to develop an invasive cancer if left untreated. In women with lower grade lesions (grades I and II) the rate of development of subsequent invasive cancer is lower. At present patients likely to progress to invasive tumour cannot be reliably detected, so ideally, from the point of view of preventing an invasive tumour, all patients should be referred for further investigation and treatment. This has enormous resource implications and also causes stress and anxiety to the patients, many of whom may not have gone on to develop an invasive carcinoma.

Cytology may be the best way of detecting cervical intraepithelial neoplasia but is not adequate for its evaluation. Most centres in Britain use colposcopy for evaluating neoplasia and for deciding on suitability of treatment with ablative procedures such as cryosurgery, laser, diathermy, cold coagulation, or cone biopsy. Ablation is successful in at least $80 \%$ of women and those having an abnormal smear test result after ablation have a 25 -fold increased risk of developing an invasive tumour. Further treatment will need to be planned taking into account younger women's reproductive desires; older women may not find the alternative to further ablation, hysterectomy, more acceptable. Thorough discussion of the pros and cons are needed-repeated ablation carries its own risks, including cervical stenosis and failure to prevent an invasive tumour developing. Long term follow up is mandatory in all cases.

\section{TREATMENT OF INVASIVE CANCER}

Early invasive cervical carcinoma (stage Ib and IIa, table II) is treated equally well by radical hysterectomy or radiotherapy though the pattern of side effects varies. ${ }^{18} 19$ For more advanced disease (stages IIb-IVa) radiotherapy is generally the best treatment, though some centres will also undertake surgery in selected patients after radiation. Many patients are treated with intracavitary radioactive isotopes which deliver a very high dose to the central pelvis. The isotope is inserted under general anaesthesia, and the recent introduction of remote afterloading machines has considerably reduced treatment times and exposure of staff to irradiation. Further external beam radiotherapy is usually given to treat the pelvic lymph nodes.

Success rates decrease with increasing tumour bulk and stage (table III). Very advanced or recurrent disease, which is not treatable by irradiation, and previously irradiated tumours have been managed recently by combinations of cytotoxic drugs based on cisplatin. Though such treatment is not curative high objective response rates $(50-60 \%)$ have been reported. ${ }^{21}$ Because of this some units have tried using chemo-

TABLE II-Randomised clinical trials producing five year survival data for management of operable cervical carcinoma (stages Ib, IIa)

\begin{tabular}{llccc}
\hline & & & \multicolumn{2}{c}{5 Year survival (\%) } \\
\cline { 4 - 5 } Trial & Stage & $\begin{array}{c}\text { No of } \\
\text { patients }\end{array}$ & Radiation & Surgery \\
\hline Cullhed $^{14}$ & Ib, IIa & 310 & 75 & 86 \\
Morley and Seski" $^{\text {Newton }}$ & I & 401 & 83 & 87 \\
Perez $^{10}$ & I & 119 & 74 & 81 \\
& I & 78 & 88 & 82 \\
& IIa & 24 & 71 & 57 \\
\hline
\end{tabular}

TABLE III-Carcinoma of cervix: survival by stage of disease (results for $1969-72)^{20}$

\begin{tabular}{lcc}
\hline & No of patients treated & No(\%) surviving at 5 years \\
\hline Stage I & 12452 & $9961(80 \cdot 0)$ \\
Stage II & 13583 & $8005(58 \cdot 9)$ \\
Stage III & 10531 & $3287(31 \cdot 2)$ \\
Stage IV & 1841 & $153(8 \cdot 3)$ \\
Not staged & 54 & $24(44 \cdot 4)$ \\
\hline Total & 38461 & $21430(55 \cdot 7)$ \\
\hline
\end{tabular}


therapy as part of the primary management. So far it is not clear if this approach of adjuvant chemotherapy will prolong survival of patients with cervical cancer as has been shown in premenopausal women with breast cancer. ${ }^{22}$ Most researchers using this approach have given chemotherapy before irradiation in an attempt to shrink the tumour and to eradicate microscopic metastases.

Randomised trials testing adjuvant chemotherapy have been small and past experience, primarily in the adjuvant treatment of breast cancer, has clearly shown that large numbers of patients are needed before the success or otherwise of adjuvant treatment can be judged. The Medical Research Council is coordinating a series of randomised clinical trials testing the role of chemotherapy before irradiation or surgery, or both, (so called neoadjuvant treatment) in patients with cervical carcinoma considered to be at risk of relapse based on tumour bulk or stage. The council is also gathering data from similar trials taking place worldwide to undertake a prospective meta-analysis. Until there are data to show that it improves survival adjuvant chemotherapy for cervical cancer should be regarded as experimental.

\section{SUMMARY}

Death rates from cervical cancer have already fallen this century and for patients with invasive cervical cancer five year survival rates are greater than for most solid tumours. Better screening for premalignant changes may further reduce the incidence of invasive cancer; indeed, it has been claimed that the reduction in mortality could be as high as $90 \%$, though estimates of screening efficacy have varied greatly. For those with advanced invasive carcinoma neoadjuvant chemotherapy may reduce the risk of relapse and improve survival.

1 Mack TM, Cozen W, Quinn MA. Epidemiology of cancer of the endometrium, ovary, and vagina. In: Coppleson M, ed. Gynecologic oncology. New York: Churchill Livingstone, 1992
2 Lynch HT, Bewtra C, Lynch JF. Familial ovarian carcinoma. Clinical nuances. Am $\mathcal{Y}$ Med 1986;81:1073-6.

3 Ponder BA. Genetic predisposition to cancer. Br $\mathcal{C}$ Cancer 1991;64:203-4.

4 Cuckle HS, Wald NJ. The evaluation of screening tests for ovarian cancer. In Ovarian cancer: biological and therapeutic challenges. London: Chapman and
Hall Medical, 1990:229-39.

5 Richardson GS, Scully RE, Ni N, Nelson JH. Common epithelial cancer of the ovary. N Engl F Med 1985;312:415-24.

6 Heintz AP, Van Oosterom AT, Trimbos JB, Schaberg A, Van der Velde E Nooy $M$. The treatment of ovarian carcinoma. I. Clinical variables associated with prognosis. Gynecol Oncol 1988;30:347-58.

7 Pickel. Intraperitoneal and retroperitoneal spread of ovarian cancer In: Sharp F, Mason W, Leake R, eds. Ovarian cancer: biological and therapeutic challenges. London: Chapman and Hall, 1990.

8 Dembo AJ, Bush RS, Beale F, Bean H, Pringle J, Sturgeon J. The Princess Margaret Hospital study of ovarian cancer: stage I, II and asymptomatic III presentations. Cancer Treatment Reports 1979;63:249-56.

9 Smith JP, Rutledge FN, Declos L. Postoperative treatment of early cancer of the ovary: a random trial between post operative irradiation and chemotherapy. National Cancer Institute Monograms 1975;42:149-53.

10 Morrow CP. In: Coppleson M, ed. Gynecologic oncology. New York: Churchill Livingstone, 1992:897.

11 Advanced Ovarian Cancer Trialists Group. Chemotherapy in advanced ovarian cancer: an overview of randomised clinical trials. BMF 1991;303 884-93.

$12 \mathrm{Ng}$ ABP. Diagnostic cytopathology. In: Coppleson M, ed. Gynecologic oncology. New York: Churchill Livingstone, 1992:277-96.

13 Coppleson M, Atkinson KH, Dalrymple JC. Cervical squamous and glandula intraepithelial neoplasm: clinical features and review of management. In: Coppleson M, ed. Gynecologic oncology. New York: Churchill Livingstone, 1992:571-607.

4 Cullhed S. Carcinoma cervicis uteri stages I and IIa. Acta Obstet Gynecol Scand 1978;suppl 78:1-149.

15 Morley G, Seski J. Radical pelvic surgery versus radiation therapy for stage carcinoma of the cervix (exclusive of microinvasion). Am $\mathcal{f}$ Obstet Gynecol 1976;126:785-96.

16 Newton $M$. Radical hysterectomy or radiotherapy for stage I cervical cancer. A prospective comparison with 5 and 10 years follow-up. $A m \mathcal{F}$ Obstet Gynecol 1975;123:534-42.

17 Perez CA, Camel HM, Kao MS, Askin F. Randomised study of preoperative radiation and surgery or irradiation alone in treatment of stage Ib and II radiation and surgery or irradiation alone in treatment

18 Averett HE, La Platney DR, Little WA. Current role of radical hysterectomy as primary therapy for invasive carcinoma of the cervix. Am 7 Obstet Gymecol 1969;105:79-86.

19 Jampolis S, Andras J, Fletcher GH. Analysis of sites and causes of failure of irradiation in invasive squamous cell carcinoma of the intact uterine cervix. Radiology 1975;115:681-5.

20 International Federation of Gynaecology and Obstetrics. Seventeenth annual report. Radiumhemmet Stockholm: IFGO, 1977.

21 Buxton EJ, Meanwell CA, Hilton C, Mould JJ, Spooner D, Chetiyawardon $A$, et al. Combination bleomycin, ifosfamide, and cisplatin chemotherapy in cervical cancer. $f$ Natl Cancer Inst 1989;81:359-61.

22 Early Breast Cancer Trialists Collaborative Group. Systemic treatment of early breast cancer by hormonal, cytotoxic, or immune therapy. Lancet 1992; 339:1-14, 71-84.

\section{OBITUARY}

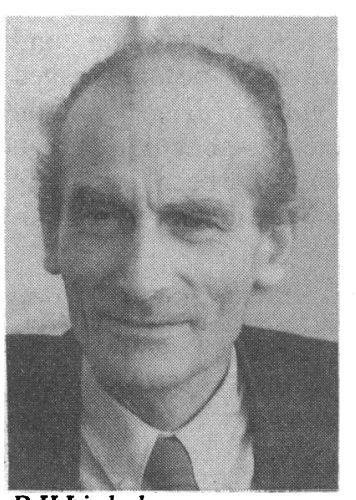

$R H$ Lindenbaum

\section{R H LINDENBAUM}

FRCP

Clinical genetics is a relatively new specialty, and Dick Lindenbaum was one of its first full time practitioners. His skill and dedication did much to persuade colleagues in more established specialties of the clinical value of a consultant geneticist.

Dick had boundless enthusiasm and curiosity: he was widely travelled, active (a keen walker and mountain biker), and an accomplished linguist. $\mathrm{He}$ wrote over 80 research papers on such disparate topics as chromosomal malsegregation, dysmorphology, muscular dystrophy, neurocutaneous disorders, and prenatal diagnosis. His hallmarks were acute observation and meticulous clinical descriptionthough he was (self confessedly) one of the world's worst administrators. Above all, he was a consummate clinician. His skill in recognising rare and obscure syndromes was matched by his helpfulness. He was particularly interested in patient support organisations and was among the first to perceive their value.

Dick Lindenbaum was extraordinarily knowledgeable and wise, being a source of sound advice to patients and colleagues. He died of cancer, a few months after the death of his brother. - M BOBROw

Richard Herbert Lindenbaum, consultant clinical geneticist to Oxford Area Health Authority since 1974, died 10 March aged 59. Born 9 fuly 1933; educated St Paul's School and University
College Oxford and St Mary's Hospital, London (BM, BCh 1959). Senior registrar at Westminster and Westminster Children's Hospitals; lecturer in clinical genetics at Medical Research Council Population Genetics Units, Oxford, 1970-4.

\section{J S TOMKINSON \\ CBE, FRCS, FRCOG}

As secretary general of the International Federation of Gynaecology and Obstetrics from 1976 to 1985 John Tomkinson became known internationally and was made an honorary fellow of gynaecological societies in 12 countries. He was awarded the Copernicus medal by the Academy of Medicine in Cracow and was the only British member of the Continental Gynaecological Club of America.

John was appointed a consultant at Guy's Hospital and Queen Charlotte's Maternity Hospital in 1953 and at the Chelsea Hospital for Women in 1971. He was consultant adviser in obstetrics and gynaecology to the Department of Health and Social Security from 1966 to 1981 and a coauthor of five of the reports on confidential enquiries into maternal death in England and Wales (1964-78); he used the material from these publications for lectures on maternal death. He was also a member of the council of the Royal College of Obstetricians and Gynaecologists (1960-71), a coopted member of the council of the Royal College of Surgeons, and deputy chairman of the Central Midwives Board. 